\section{FRI0163 INVESTIGATION OF THE PERIODONTAL CONDITION AMONG RHEUMATOID ARTHRITIS (RA) PATIENTS AND ANALYSIS OF INFLAMMATORY MEDIATOR IN THEIR SERUM AND GINGIVAL CREVICULAR FLUID (GCF)}

F. Xiao, P. Zhang, X. Li, Y. Mou, H. Chen, Z. Chen. The Third Affiliated Hospital of Sun Yat-Sen University, Guangzhou, China

Background: Rheumatoid arthritis (RA) and periodontitis (PD) are common chronic inflammatory diseases with remarkable pathological and clinical similarities.A lot of similarities exist between RA and PD at cellular and molecular levels.

Objectives: To analyse the PD incidence among RA patients, measure the level

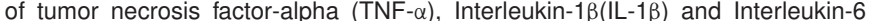
(IL-6)in their serum and gingival crevicular fluid (GCF), and to investigate their relationship.

Methods: The experimental group was composed of 350 patients with RA and the control group consisted of 426 age and gender matched healthy individuals, check and record periodontal condition by dentist,analyse the PD incidence of the two groups, then select 64 PD patients without systematic diseases and 47 PD patients with a stable condition of RA, Detect and compare their periodontal status and the level s of TNF- $\alpha, \mathrm{IL}-1 \beta$ and IL- 6 in their serum and GCF.

Results: The percentage of PD was $67.7 \%$,which was statistically significant higher than control group $(43.6 \%)(P<0.001)$. The periodontal disease index (PDI),Probing depth (PD)and Clinical attachment loss (CAL) in patients with $\mathrm{PD}$ and $\mathrm{RA}$ are all significantly higher than those with simple $\mathrm{PD}(\mathrm{P}<0.05)$. But the bleeding on probing (BOP) in the two groups was not statistically significant $(P>0.05)$. Meanwhile, the level of TNF- $\alpha, \mathrm{IL}-1 \beta$ and IL-6 in serum and GCF are significantly higher in all PD patients than oral healthy individuals $(P<0.01)$. Although those inflammatory mediators in serum are much higher in patients with $P D$ and $R A$ than in those with simple $P D(P<0.01)$, there is no difference in the two group about those inflammatory mediators in GCF. The serum level of TNF- $\alpha, \mathrm{IL}-1 \beta$ and IL- 6 in patients with PD and RA are positively correlated with the corresponding inflammatory mediators in their GCF ( $r$ value was $0.531,0.422$ and 0.770 respectively,p $<0.01$ ).

Conclusions: Individuals with RA are more likely to experience periodontitis compares to healthy subjects, and the periodontitis is much more serious in patients with RA and PD than those with simple PD. the serum and GCF inflammatory factors' level increased significantly in patients with RA and PD,even during their RA stable period. The serum level of inflammatory factors in patients with $\mathrm{PD}$ and RA are positively correlated with the corresponding inflammatory mediators in their GCF. There is correlation between periodontitis and RA, and they may be the risk factors for eachother, the improvement of the periodontal status in patients with RA and PD may help to control systemic inflammation level and to make comprehensive treatment measure for RA patients.

References:

[1] Venkataraman A, Almas K.Rheumatoid Arthritis and Periodontal Disease. An Update.N Y State Dent J. 2015 Aug-Sep;81(5):30-6.

[2] Araújo VM, Melo IM, Lima V.Relationship between Periodontitis and Rheumatoid Arthritis: Review of the Literature.Mediators Inflamm. 2015;2015:259074. doi: 10.1155/2015/259074. Epub 2015 Aug 4.

[3] Javed F, Ahmed HB, Mikami T, Almas K, Romanos GE, Al-Hezaimi K.Cytokine profile in the gingival crevicular fluid of rheumatoid arthritis patients with chronic periodontitis.J Investig Clin Dent. 2014 Feb;5(1):1-8. doi: 10.1111/jicd.12066. Epub 2013 Dec 20.

Disclosure of Interest: None declared

DOI: 10.1136/annrheumdis-2017-eular.5919

\section{FRI0164 DIFFERENCES BETWEEN RA PATIENTS WITH AND WITHOUT ILD FROM A UNITED STATES TERTIARY REFERRAL CENTER}

R. Meehan $^{1}$, J. Solomon ${ }^{1}$, J. Crooks ${ }^{1}$, D. Muram ${ }^{2}$, E. Hoffman ${ }^{1}$, P. Zelarney ${ }^{1}$. ${ }^{1}$ National Jewish Health, Denver; ${ }^{2}$ Eli Lilly and Company, Indianapolis, United States

Objectives: To describe the characteristics of RA patients with and without ILD and to determine if medication use constitutes a risk factor for the development of ILD.

Methods: The medical records of RA patients with and without ILD treated at one academic center between 2008 and 2016 were analyzed. Data extracted include: patient demographics, serology, medication use (prednisone, DMARDs, biologics or small molecule drugs), and self-reported disease activity as MDHAQ and RAPID 3 scores. The subtype of ILD was based upon HRCT imaging, and some patients through histology. Differences between RA-ILD patients and RA patients without ILD were determined by Fishers exact test, and t-tests with a $\mathrm{p}<0.05$ were considered statistically significant.

Results: The demographics and clinical data of 1,024 RA non-ILD patients and 96 RA-ILD patients indicate ILD patients were older males, had a higher mortality and were less likely to have been never smokers (Table 1). At the onset of ILD diagnosis, $31 \%$ were on no medication, $56 \%$ on monotherapy (MTX $16 \%$, prednisone $13 \%$, Etanercept $7 \%$, adalimumab or LEF $5 \%$, Rituximab $4 \%$, HCQ $3 \%$, and $<1 \%$ on infliximab, SSZ or tofacitinib) and $11 \%$ received combination therapy. Twenty five percent of the RA-ILD patients developed ILD preceding or coinciding with the diagnosis of RA. After the onset of ILD those patients were more likely to receive prednisone and less likely to receive MTX than those without ILD $(p<0.05)$. The predominant types of ILD were as follows: UIP $27 \%$, NSIP $15 \%$, NSIP vs UIP $14 \%$, and unclassifiable $9 \%$.

Table 1

\begin{tabular}{lccc}
\hline & RA-ILD & RA-without ILD & P values \\
\hline Total number of patients & 96 & 1,024 & \\
Gender (Male) & $48(50 \%)$ & $238(23 \%)$ & $<0.001$ \\
Age $>65$ & $57(59 \%)$ & $337(33 \%)$ & $<0.001$ \\
Never smoker & $33(34 \%)$ & $504(50 \%)$ & $<0.001$ \\
Fatalities & $15(16 \%)$ & $38(4 \%)$ & $<0.001$ \\
BMI $(>25)$ & $64(67 \%)$ & $671(66 \%)$ & NS \\
RF+ $(>14$ units) & $67(74 \%)$ & $678(69 \%)$ & NS \\
CCP+ $(>20$ units) & $62(65 \%)$ & $617(61 \%)$ & NS \\
MDHAQ & 0.7 & 0.7 & NS \\
Rapid3 & 10.6 & 12.0 & NS \\
\hline
\end{tabular}

Conclusions: RA-ILD patients differed from RA patients without ILD in demographic characteristics, but not in self-reported measures of disease activity or serology. Thirty one percent of RA-ILD patients were not on any immunomodulatory medications at the time of ILD diagnosis, and $25 \%$ of RA-ILD patients developed ILD preceding, or coinciding with, the onset of active RA. Our results did not identify a specific drug class or biologic agent as a risk factor for developing ILD in RA patients.

Disclosure of Interest: R. Meehan Grant/research support from: Eli Lilly and Company, J. Solomon: None declared, J. Crooks: None declared, D. Muram Employee of: Eli Lilly and Company, E. Hoffman: None declared, P. Zelarney: None declared

DOI: 10.1136/annrheumdis-2017-eular.4134

\section{FRI0165 SERUM FREE LIGHT CHAINS OF IMMUNOGLOBULINS IN RHEUMATOID ARTHRITIS: CORRELATION WITH INTERSTITIAL LUNG DISEASE}

S. Belghali ${ }^{1}$, S. Lataoui ${ }^{1}$, S. Ferchichi ${ }^{2}$, K. Baccouche ${ }^{1}$, N. Elamri ${ }^{1}$, K. Limam ${ }^{2}$, H. Zeglaoui ${ }^{1}$, E. Bouajina ${ }^{1} .{ }^{1}$ Rheumatology Department; ${ }^{2}$ Laboratory of Biochemistry, Farhat Hached Hospital, Sousse, Tunisia

Background: Lungs in Rheumatoid arthritis (RA) seem to play an important role, not only as an extra-articular manifestation but also as a site of initiation of the disease and activation for the immune response. B lymphocytes are thought to be involved in the pathophysiology of RA and interstitial lung disease (ILD). The encouraging results of the use of Anti-CD20 treatments to stabilize articular symptoms and lung involvement reinforce this theory. Serum Free immunoglobulin Light Chains (FLC) might represent, in this context, an interesting marker of B lymphocytes activation during this disease.

Objectives: To determine the serum FLC levels in RA patients with and without ILD and to study their possible association with disease characteristics and activity.

Methods: Fifteen RA patients with clinically symptomatic interstitial lung disease, confirmed by computed tomography (RA-ILD) $(53.3 \%$ females; mean age $61,27 \pm 6,48$ years) and age matched RA patients $(66.7 \%$ females) with no clinically evident interstitial disease (non-ILD) were studied. Clinical and immunological inflammatory characteristics were assessed for all the patients. FLC levels were quantified by turbidimetry (Freelite TM Kappa and Lambda Kits, The Binding Site, UK).

Results: The mean serum FLC-к levels (RA-ILD: $40,74 \pm 16,94 \mathrm{ng} / \mathrm{ml}$ vs non-ILD $24,88 \pm 8,87 \mathrm{ng} / \mathrm{ml}, \mathrm{p}=0,003$ ) and FLC- $\lambda$ levels (RA-ILD: $37,34 \pm 16,56 \mathrm{ng} / \mathrm{ml}$ vs non-ILD $26,28 \pm 7,22 \mathrm{ng} / \mathrm{ml}, \mathrm{p}=0,028$ ) were significantly higher in patients with ILD compared to non-ILD, while the serum FLC-ratiok/ $\lambda$ (RA-ILD: $1,15 \pm 0,38$ vs non-ILD $1,00 \pm 0,43, p=0,304$ ) were comparable.

There was no significant difference for the DAS28 disease activity score between both groups (RA-ILD: $4,6 \pm 1,5$ vs non-ILD 4,38 $\pm 1,46, p=0,69$ ). No significant correlations were found between the DAS28-score and FLCK, FLC $\lambda$ and FLCratiok $/ \lambda(p>0,05)$. FLC-к levels correlated significantly with ESR and CRP levels $(p<0.001)$, FLC- $\lambda$ levels with CRP $(p=0,005)$ but not with ESR $(p=0,247)$ and FLC-ratiok/ $\lambda$ did not correlate with both ESR and CRP levels.

Conclusions: High levels of serum FLC are associated with RA-ILD and with a higher degree of inflammation, supporting the role of $B$ cell activation in the pathophysiology of RA with ILD.

Disclosure of Interest: None declared

DOI: 10.1136/annrheumdis-2017-eular.6364

\section{FRI0166 RADIOGRAPHIC STRUCTURAL DAMAGE IS WORSE IN THE DOMINANT THAN THE NON-DOMINANT UPPER EXTREMITY IN PATIENTS WITH RHEUMATOID ARTHRITIS}

S. Nakazaki ${ }^{1}$, T. Murayama ${ }^{1}$, S. Kato ${ }^{2} .{ }^{1}$ Rheumatology, Johoku Hospital;

${ }^{2}$ Rheumatology, Kamiaraya Clinic, Kanazawa, Japan

Background: The relationship between mechanical stress and radiographic damage in rheumatoid arthritis (RA) has been detected in patients with hemiplegia ${ }^{1)}$ or poliomyelitis ${ }^{2}$. In patients without neurological disorder, Koh reported that radiographic damage was worse and progressed faster in the dominant hand in 
individuals with early $\mathrm{RA}^{3}$ ) . However, few studies have detected the radiographic damage in the whole upper extremity on the mechanical stress.

Objectives: To examine the relationship between mechanical stress and radiographic damage in RA, we evaluated the joint destruction of the dominant and non-dominant upper extremity.

Methods: The joint destruction of the upper extremity (shoulder, elbow, wrist, metacarpo-phalangeal, interphalangeal, proximal interphalangeal) in 295 patients with RA, who were from 25 years to 91 years (mean age 64 years, mean disease duration 183 months, $86 \%$ females and $97 \%$ right-handed), was assessed according to the eroded joint, which was defined as $>2$ by Larsen scores for radiographic damage. These were divided into the dominant and non-dominant upper extremity. The Wilcoxon signed-rank test was used to examine the difference between the eroded joint count $(\mathrm{EJC})$ in the dominant and the non-dominant upper extremity.

Results: The mean EJC in the dominant and the non-dominant upper extremity was 2.4 and 2.05 respectively. The EJC was significantly more in the dominant than the non-dominant upper extremity. And, in regards to every joint of upper extremity, the eroded joint rate was higher in the dominant than the non-dominant

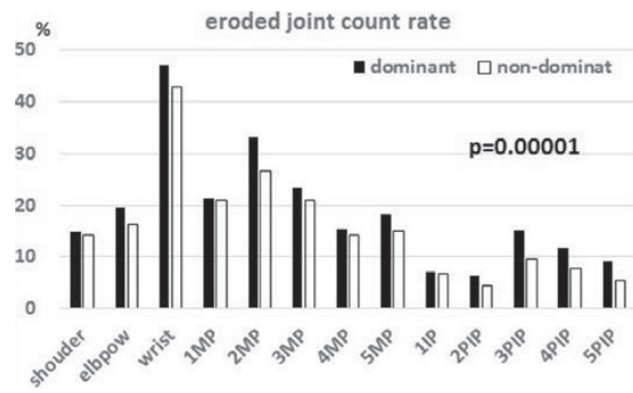

Conclusions: The eroded joint count of the upper extremity was significantly more in the dominant than the non-dominant one, therefore it was suggested that the mechanical stress influenced the radiographic damage in patients with $R A$ References:

[1] John $\mathrm{H}$ Bland, et al.: Hemiplegia and rheumatoid hemiarthritis. Arthritis Rheum (11) 1:72-80, 1968

[2] E N Glick, et al.: Asymmetrical rheumatoid arthritis after poliomyelitis. Br Med J 3: 26-28, 1967.

[3] Jung Hee Koh, et al.: Radiographic structural damage is worse in the dominant than the non-dominant hand in individuals with early rheumatoid arthritis. PLOS ONE, doi: 10. 1371/journal. Phone.0135409 August 6, 2015.

Disclosure of Interest: None declared

DOI: 10.1136/annrheumdis-2017-eular.3621

\section{FRI0167 COMPARATIVE CARDIOVASCULAR SAFETY OF ABATACEPT AND TUMOR NECROSIS FACTOR INHIBITORS IN RHEUMATOID ARTHRITIS PATIENTS WITH AND WITHOUT CARDIOVASCULAR DISEASE: A POPULATION-BASED COHORT STUDY}

Y. Jin ${ }^{1}$, E.H. Kang ${ }^{1,2}$, G. Brill ${ }^{1}$, R.J. Desai ${ }^{1}$, S.C. Kim ${ }^{1,3} \cdot{ }^{1}$ Division of Pharmacoepidemiology and Pharmacoeconomics, Brigham and Women's Hospital, Harvard Medical School, Boston, United States; ${ }^{2}$ Division of Rheumatology Department of Internal Medicine, Seoul National University Bundang Hospital, Seongnam, Korea, Republic Of; ${ }^{3}$ Division of Rheumatology, Immunology and Allergy, Brigham and Women's Hospital, Harvard Medical School, Boston, United States

Background: Rheumatoid arthritis (RA) patients have an increased risk of cardiovascular disease (CVD). Recent studies suggested that treatment of RA with tumor necrosis inhibitors (TNFi) can reduce the risk of cardiovascular events. However, it is unclear how abatacept, a selective costimulation modulator, affects cardiovascular risk among RA patients compared with TNFi.

Objectives: To evaluate the comparative cardiovascular safety of abatacept versus TNFi in RA patients with and without underlying CVD.

Methods: We identified RA patients aged $\geq 18$ with $\geq 2$ RA ICD-9 codes (714.xx) separated by $>7$ days but $<365$ days, from two large insurance claims data across the U.S.: Medicare (2008-2013) and Truven MarketScan (2006-2015). Only new users of abatacept or TNFi (adalimumab, etanercept, certolizumab, golimumab, and infliximab) were included. The primary outcome of interest was a composite endpoint of CVD including myocardial infarction (MI), stroke/transient ischemic stroke, or coronary revascularization. Secondary outcomes were each component of the primary outcome, incident heart failure (HF), and venous thromboembolism (VTE). 1:1 propensity score (PS) matching was performed separately in each database and each subgroups (with or without baseline CVD). Then the PS-matched subgroups were aggregated to form the overall matched cohort. Cox regression model was used to estimate the hazard ratio $(\mathrm{HR})$ and $95 \%$ confidence interval $(\mathrm{Cl})$ of risk of each outcome. Estimates from two different databases were combined through an inverse variance-weighted fixed-effects model.

Results: After 1:1 PS matching, there were 6,102 patient pairs from Medicare and 6.934 pairs from MarketScan. Among them, patients with baseline CVD were $35.3 \%$ in Medicare and $14.0 \%$ in MarketScan. Baseline characteristics were well balanced between two treatment groups after matching (standardized mean difference $<0.1$ ). In Medicare cohort, abatacept consistently showed a decreased risk of composite CVD compared with TNFi in overall and each subgroup (Figure). However, in MarketScan cohort, where the population was younger than Medicare cohort, there was no association between abatacept and CVD compared to TNFi. After combining the two databases, abatacept was significantly associated with reduced risk of composite CVD outcome vs. TNFi in overall cohort ( $\mathrm{HR}=0.79$, 95\% $\mathrm{Cl}=0.67-0.92$ ) and baseline CVD subgroup ( $\mathrm{HR}=0.79,95 \% \mathrm{Cl}=0.64-0.98$ ). We also observed similar trend for secondary outcomes, where abatacept had decreased risk than TNFi.

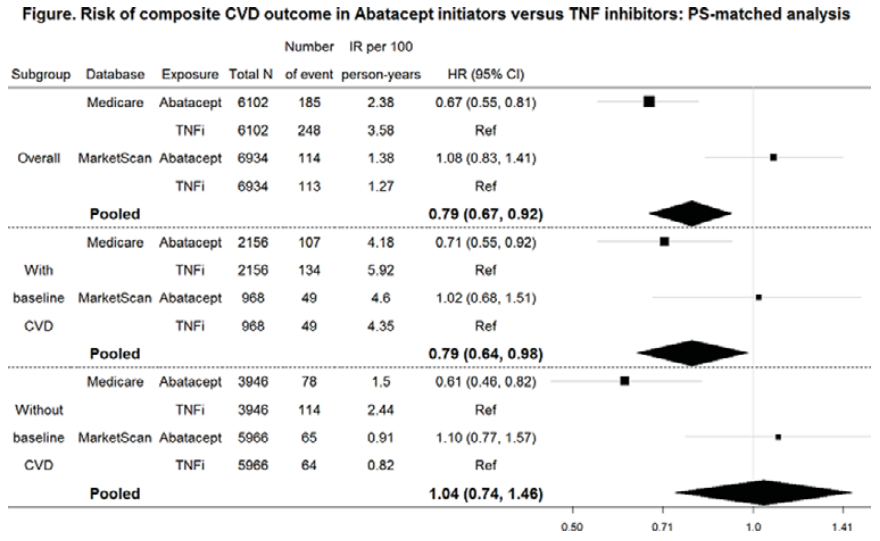

Conclusions: In this large multi-database population-based study of RA patients abatacept treatment was associated with reduced risk of CVD compared to TNFi, especially among older population and patients with prior CVD conditions.

Disclosure of Interest: None declared

DOI: 10.1136/annrheumdis-2017-eular.2727

\section{FRI0168 RESTORATION OF DECREASED LYMPHOCYTES, CD8+T CELL SUBSETS WITH TH1 SKEWED PHENOTYPE ASSOCIATE WITH SPONTANEOUS REGRESSION OF LYMPHO-PROLIFERATIVE DISORDERS IN RHEUMATOID ARTHRITIS PATIENTS TREATED WITH METHOTREXATE}

S. Saito ${ }^{1}$, K. Suzuki ${ }^{1}$, K. Yamaoka ${ }^{1}$, K. Amano ${ }^{2}$, M. Tokuhira ${ }^{3}$, T. Takeuchi ${ }^{1}$. ${ }^{1}$ Division of Rheumatology, Department of Internal Medicine, Keio University School of Medicine, Tokyo; ${ }^{2}$ Division of Rheumatology, Saitama Medical Center, Saitama Medical University; ${ }^{3}$ Division of Hematology, Saitama Medical Center, Saitama Medical University, Saitama, Japan

Background: Lympho-proliferative disorder (LPD) is known as a relatively rare but life-threatening complication in RA patients under MTX administration. Spontaneous regression of LPD after MTX withdrawal is regarded as a distinct character of LPD under MTX administration. Previous study from our institution [1] and others [2] reported the link between decreased lymphocyte counts at LPD diagnosis and restoration after MTX cessation and the regression of LPD.

Objectives: To investigate the immunological factors including lymphocyte sub sets which involved in spontaneous regression of LPD following MTX withdrawal. Methods: We studied 35 RA patients complicated with LPD under MTX administration in our institution. Age, sex, RA disease duration matched contro MTX-treated patients $(\mathrm{N}=35)$ were selected. LPD patients were divided into two groups regarding to the status of LPD after MTX cessation; regressive group $(\mathrm{N}=22)$ and persistent group $(\mathrm{N}=13)$. Clinical features were compared among 3 groups. Flowcytometric analysis of the whole blood sample and measurement of cytokine concentration in ELISA were conducted in a part of the LPD patients $(\mathrm{N}=10,7$ regressive, and 3 persistent LPD) and controls $(\mathrm{N}=10)$. The time of MTX cessation, which was simultaneous with LPD diagnosis, was defined as week 0 and blood sample was collected at week 0,4 and 12 .

Results: At week 0 , number of peripheral lymphocytes was significantly decreased in regressive group, compared to persistent group and control group. Flowcytometric analysis revealed significant decrease in proportion of effector memory CD8+ $T$ cells (EMCD8+T), Epstein Barr Virus specific CD8+ $T$ cells (EBV specific CD8+) and T helper 1 cells (Th1 cells) subset in regressive group compared to control group. Following MTX cessation, significant increase in proportion and absolute number of these subsets were observed only in the regressive group, but not in persistent group. Expansion of Th1 cells and EMCD8+ $\mathrm{T}$ cells significantly correlated with increase of serum IFN- $\gamma$, and expansion of EMCD8+ T cells inversely correlated with change of serum IL-15.

Conclusions: Restoration in proportion and absolute number of Th1 cells, EMCD8+T cells and EBV specific CD8+ T cells coincided with increase of IFN- $\gamma$ and associated with regression of LPD developed under MTX administration Since changes of those immunological factors were not observed in persistent LPD, this study would be the first report to demonstrate the difference of 